\title{
CORRIGENDUM
}

\section{A Pilot Investigation of Cognitive Behavioural Therapy for Clinical Perfectionism in Obsessive Compulsive Disorder-CORRIGENDUM}

\author{
S. K. Sadri, R. A. Anderson, P. M. McEvoy, R. T. Kane and S. J. Egan
}

doi: https://doi.org/10.1017/S1352465816000618 Published online: 31 January 2017

Values that were incorrectly reported have been corrected in Table 1 (below) and corresponding sections of the article as follows (all changes are italicised and underlined):

1. Results (page 316) states "five $(45.5 \%, \mathrm{CM})$, two $(18.2 \%, \mathrm{PS})$ and one $(9.1 \%, \mathrm{CPQ})$ participant in the intervention group achieved a reliable reduction on the perfectionism measures" should read "five (45.5\%, CM), three $(27.3 \%, P S)$ and one (9.1\%, CPQ) participant in the intervention group achieved a reliable reduction on the perfectionism measures".

"The Phi coefficient indicated moderate associations for $\mathrm{CM}$ and $\mathrm{CPQ}$, and a small-to-moderate association for PS" should read "The Phi coefficient indicated moderate associations for the CM, $P S$, and $C P Q$ ".

2. Results (page 318) states: "According to these criteria, $45.5 \%$ of the intervention group were recovered, with the remainder unchanged" should read "According to these criteria, $27.3 \%$ of the intervention group were recovered, two were improved, and six were unchanged".

Discussion (page 318) states: “...45.5\% of participants experienced a clinically significant improvement in OCD severity should read "...27.3\% of participants experienced a clinically significant improvement in OCD severity”.

\section{Reference}

Sadri, S. K., Anderson, R. A., McEvoy, P. M., Kane, R. T. and Egan, S. J. (2017). A pilot investigation of cognitive behavioural therapy for clinical perfectionism in obsessive compulsive disorder. Behavioural and Cognitive Psychotherapy, 45, 312-320. doi: https://doi.org/10.1017/ S1352465816000618 
Table 1. Comparison of effect sizes and proportion of participants in the waitlist and intervention conditions demonstrating reliable and clinically significant change on outcome variables from pre to post treatment

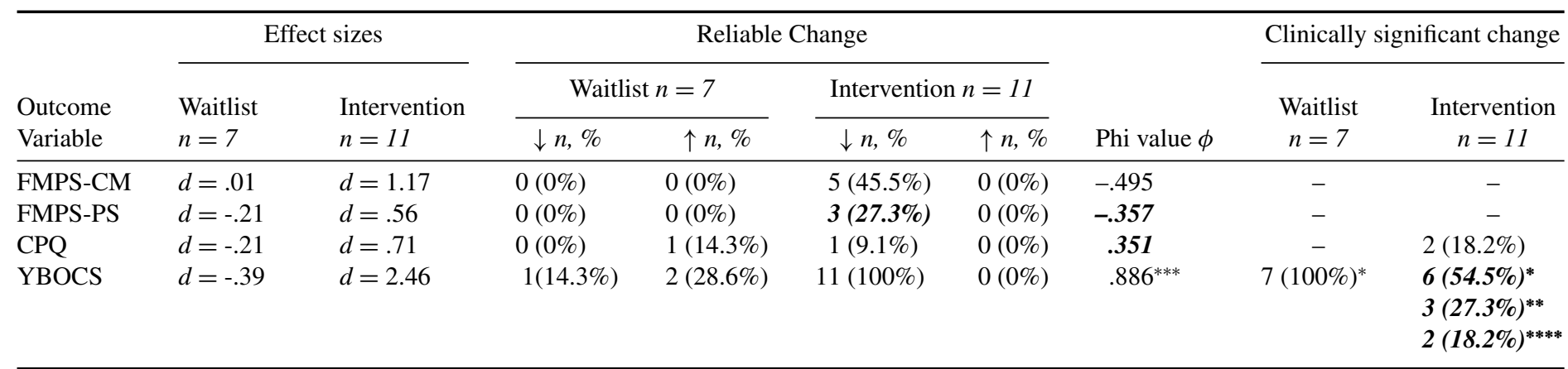

Note: The intervention group includes data from immediate intervention and waitlist participants; $n$, $\%=\downarrow$ number and percentage of participants who experienced a reliable decrease (improvement) on the outcome variable; $\uparrow n$, $\%=$ number and percentage of participants who experienced a reliable increase (deterioration) on the outcome variable; FMPS = Frost Multidimensional Perfectionism Scale; CM = Concern Over Mistakes; $\mathrm{PS}=$ Personal Standards $; \mathrm{CPQ}=$ Clinical Perfectionism Questionnaire; YBOCS $=$ Yale Brown Obsessive Compulsive Personality Scale ${ }^{*}=$ unchanged; ${ }^{* *}=$ recovered; ${ }^{* * *}$ improved; $d=$ Cohen's magnitude of effect; Phi value $=$ magnitude of effect $(.10=$ small, $.30=$ moderate, $+.50=$ large); ${ }^{* * *}$ strong positive association. Corrected values are bolded and italicised. 